Mathematical Research Letters 2, 643-651 (1995)

\title{
LATTICES AND CODES WITH LONG SHADOWS
}

\author{
NOAM D. ELKIES
}

\section{Introduction}

By a characteristic vector of an integral unimodular lattice $L \subset \mathbf{R}^{n}$ we mean a vector $w \in L$ such that $(v, w) \equiv(v, v) \bmod 2$ for all $v \in L$. Such vectors are known to constitute a coset of $2 L$ in $L$ whose norms are congruent to $n$ mod 8 (see, e.g., [Se, Ch.V]); dividing this coset by 2 yields a translate of $L$ called the shadow of $L$ in [CS2]. If $L=\mathbf{Z}^{n}$ then $w \in L$ is characteristic if and only if all its coordinates are odd, so every characteristic vector of $\mathbf{Z}^{n}$ has norm at least $n$. In [El] we proved that if $L \nsubseteq \mathbf{Z}^{n}$ then $L$ has characteristic vectors of norm $\leqslant n-8$, and described without proof all lattices for which $n-8$ is the minimum. Here we prove this result; along the way, we also obtain congruences and a lower bound on the kissing number of unimodular lattices with minimal norm 2. We then state and prove analogues of these results for self-dual codes, and relate them directly to the lattice problems via Construction A.

\section{Estimates for unimodular lattices}

Any integral lattice $L$ decomposes as the direct sum $\mathbf{Z}^{r} \oplus L_{0}$ where the $\mathbf{Z}^{r}$ is generated by the vectors of norm 1 and $L_{0}$ is a lattice of minimal norm $\geqslant 2$. (This $L_{0}$ is called the "reduced form" or "initial lattice" of $L$ in $[\mathrm{CS} 1, \mathrm{p} .414]$, the latter terminology suggesting the infinite family of lattices $L_{0}, L_{0} \oplus \mathbf{Z}, L_{0} \oplus \mathbf{Z}^{2}$, etc., of which $L_{0}$ is the initial member.) If $L$ (and thus also $L_{0}$ ) is unimodular then the shadow of $L$ is the orthogonal sum of the shadows of $\mathbf{Z}^{r}$ and $L_{0}$. Replacing $L$ by $L_{0}$ thus reduces both the rank of the lattice and the norm of its shortest characteristic vector by $r$, and does not change the difference between these two integers. We may thus restrict attention to lattices with no vectors of norm 1 for which that difference is 8 , and at the end recover all such lattices by adding arbitrarily many Z's.

Received July 13, 1995.

Partially supported by the NSF and the Packard Foundation. 
Theorem 1. Let $L$ be an integral unimodular lattice in $\mathbf{R}^{n}$ with no vectors of norm 1. Then:

i) L has at least $2 n(23-n)$ vectors of norm 2 .

ii) Equality holds if and only if $L$ has no characteristic vectors of norm $<n-8$.

iii) In that case the number of characteristic vectors of norm exactly $n-8$ is $2^{n-11} n$.

Proof. We use theta series as in [El], though here we freely invoke modular forms. For $t$ in the upper half-plane $H$ define

$$
\theta_{L}(t):=\sum_{v \in L} e^{\pi i|v|^{2} t}=\sum_{k=0}^{\infty} N_{k} e^{\pi i k t},
$$

where $N_{k}$ is the number of lattice vectors of norm $k$, and

$$
\theta_{L}^{\prime}(t):=\sum_{v \in L+\frac{w}{2}} e^{\pi i|v|^{2} t}=\sum_{k=0}^{\infty} N_{k}^{\prime} e^{\pi i k t / 4},
$$

where $w \in L$ is any characteristic vector and $N_{k}^{\prime}$ is the number of characteristic vectors of norm $k$, or equivalently the number of shadow vectors of norm $k / 4$. In [El] we noted the identity

$$
\theta_{L}\left(\frac{-1}{t}+1\right)=(t / i)^{n / 2} \theta_{L}^{\prime}(t)
$$

By a theorem of Hecke (see, e.g., [CS1, Ch.7, Thm.7]), $\theta_{L}$ is a modular form of weight $n / 2$ and can be written as a weighted-homogeneous polynomial $P_{L}\left(\theta_{\mathbf{Z}}, \theta_{E_{8}}\right)$ in the modular forms

$$
\theta_{\mathbf{Z}}(t):=1+2\left(e^{\pi i t}+e^{4 \pi i t}+e^{9 \pi i t}+\cdots\right)
$$

of weight $1 / 2$ and

$$
\theta_{E_{8}}(t)=1+240 \sum_{m=1}^{\infty} \frac{m^{3} e^{2 \pi i m t}}{1-e^{2 \pi i m t}}=1+240 e^{2 \pi i t}+2160 e^{4 \pi i t}+\cdots
$$

of weight 4 . From (3) it follows that $\theta_{L}^{\prime}$ is given by

$$
\theta_{L}^{\prime}=P_{L}\left(\theta_{\mathbf{Z}}^{\prime}, \theta_{E_{8}}\right)
$$

where

$$
\theta_{\mathbf{Z}}^{\prime}(t)=2 \sum_{m=0}^{\infty} e^{\pi i\left(m+\frac{1}{2}\right)^{2} t}=2 e^{\pi i t / 4}\left(1+e^{2 \pi i t}+e^{6 \pi i t}+e^{12 \pi i t}+\cdots\right) .
$$


We used the fact that $\theta_{E_{8}}^{\prime}=\theta_{E_{8}}$, because $E_{8}$ is an even lattice. Since $\theta_{\mathbf{Z}}^{\prime}(t) \sim 2 e^{\pi i t / 4}$ as $t \rightarrow i \infty$, while $E_{4}(i \infty)=1$ is nonzero, we see from (6) that the norm of the shortest characteristic vectors is simply the exponent of $X$ in the factorization of $P_{L}(X, Y){ }^{1}$

In our setting $N_{0}=1$ and $N_{1}=0$. We first prove part (ii) of our theorem. If $L$ has no characteristic vectors of norm $<n-8$ then $P_{L}(X, Y)$ is a linear combination of $X^{n}$ and $X^{n-8} Y$. The known values of $N_{0}, N_{1}$ determine this combination uniquely: we find that

(8) $\theta_{L}=\theta_{\mathbf{Z}}^{n}-\frac{n}{8} \theta_{\mathbf{Z}}^{n-8}\left(\theta_{\mathbf{Z}}^{8}-\theta_{E_{8}}\right)=1+0 e^{\pi i t}+2 n(23-n) e^{2 \pi i t}+\cdots$.

Thus $L$ indeed has $2 n(23-n)$ vectors of norm 2 . Conversely if $L$ is an integral unimodular lattice with $N_{1}=0$ and $N_{2} \leqslant 2 n(23-n)$ then $n<24$ and $P_{L}$ has at most 3 terms, whose coefficients are determined uniquely by $N_{0}, N_{1}, N_{2}$ :

(9)

$$
\theta_{L}=\theta_{\mathbf{Z}}^{n}-\frac{n}{8} \theta_{\mathbf{Z}}^{n-8}\left(\theta_{\mathbf{Z}}^{8}-\theta_{E_{8}}\right)+\frac{N_{2}-(2 n(23-n))}{16^{2}} \theta_{\mathbf{Z}}^{n-16}\left(\theta_{\mathbf{Z}}^{8}-\theta_{E_{8}}\right)^{2} .
$$

But then by (6) we have

$$
N_{n-16}^{\prime}=2^{n-24}\left[N_{2}-(2 n(23-n))\right] .
$$

Since $N_{n-16} \geqslant 0$ we conclude that $N_{2} \geqslant 2 n(23-n)$ even for $n<24$, as claimed in part (i) of the theorem; and equality occurs if and only if $N_{n-16}^{\prime}$ vanishes, whence the reverse implication in part (ii) follows. Finally to prove part (iii) we use $(6,8)$ to compute

$$
\theta_{L}^{\prime}=\theta_{\mathbf{Z}}^{\prime n}-\frac{n}{8} \theta_{\mathbf{Z}}^{\prime n-8}\left(\theta_{\mathbf{Z}}^{\prime 8}-\theta_{E_{8}}\right)=2^{n-11} n e^{(n-8) \pi i t}+\cdots,
$$

so $N_{n-8}^{\prime}=2^{n-11} n$ as claimed.

Fortunately the integral unimodular lattices of rank $n<24$ are completely known, and those with $N_{1}=0$ are conveniently listed with their $N_{2}$ values in the table of [CS1, pp. 416-7]. For $n<16$ the shortest characteristic vector must have norm at least $n-8$, so any unimodular lattice of minimal norm $>1$ must have $2 n(23-n)$ vectors of norm 2 ; this is confirmed by the table. When $16 \leqslant n \leqslant 23$ some lattices can have more than $2 n(23-n)$ such vectors, but it turns out there is always at least one lattice with $N_{2}=2 n(23-n)$. (Can this be proved a priori?) Thus, as

\footnotetext{
${ }^{1}$ We could now recover our theorem from [El] by observing that this exponent is at most $n$, with equality if and only if $\theta_{L}$ is proportional to $\theta_{\mathbf{Z}}^{n}$, etc.; but this is really the same proof because the crucial fact that $\theta_{\mathbf{Z}}$ vanishes at one cusp and nowhere else is also an essential ingredient of Hecke's theorem.
} 
observed by J. H. Conway, the lattices of parts (ii), (iii) of our theorem are precisely the integral unimodular lattices of rank $n<24$ with $N_{1}=0$ that minimize $N_{2}$ given $n$. As noted in [El], there are fourteen such lattices; in the following list, adapted from [El], we label them as in the table of [CS1] by the root system of norm- 2 vectors:

\begin{tabular}{c|cccccc}
$n$ & 8 & 12 & 14 & 15 & 16 & 17 \\
\hline$N_{2}$ & 240 & 264 & 252 & 240 & 224 & 204 \\
\hline & $E_{8}$ & $D_{12}$ & $E_{7}^{2}$ & $A_{15}$ & $D_{8}^{2}$ & $A_{11} E_{6}$
\end{tabular}

\begin{tabular}{c|cccccc}
$n$ & 18 & 19 & 20 & 21 & 22 & 23 \\
\hline$N_{2}$ & 180 & 152 & 120 & 84 & 44 & 0 \\
\hline & $D_{6}^{3}, A_{9}^{2}$ & $A_{7}^{2} D_{5}$ & $D_{4}^{5}, A_{5}^{4}$ & $A_{3}^{7}$ & $A_{1}^{22}$ & $O_{23}$
\end{tabular}

We noted in [El] that from our characterization of $\mathbf{Z}^{n}$ we could also recover the fact that $\mathbf{Z}^{n}$ is the only integral unimodular lattice of rank $n$ for $n<8$. Likewise from part (iii) of Theorem 1 we can recover the fact that every integral unimodular lattice of rank $n<12$ is either $\mathbf{Z}^{n}$ or $\mathbf{Z}^{n-8} \oplus E_{8}$. Indeed there would otherwise be such a lattice of rank 9,10 , or 11 with no vector of norm 1 , but then by (iii) the lattice would have $N_{n-8}^{\prime}=2^{n-11} n$, which is impossible because $N_{n-8}^{\prime}$ is an even integer for any lattice of rank $n \neq 8$.

Having obtained (10), we used $N_{n-16}^{\prime} \geq 0$ to prove $N_{2} \geqslant 2 n(23-$ $n$ ). Since $N_{n-16}^{\prime}$ is always an even integer unless $n=16$ and $\mathbf{0}$ is a characteristic vector ( $\Longleftrightarrow L$ is its own shadow $\Longleftrightarrow L$ is an even lattice), it follows that in fact

$$
N_{2} \equiv 2 n(23-n) \bmod 2^{25-n}
$$

for any even unimodular lattice with no vectors of norm 1, with the exception of the two even lattices $E_{8}^{2}, D_{16}^{+}$of rank 16 , which have $N_{2}=480$. (This is similar to the argument used in [CS1, Ch.19, p.440] to prove that there are no "extremal Type I lattices" with $16 \leqslant n \leqslant 22$, a fact which now also follows from part (i) of our Theorem.) The congruence (12) is confirmed by the table of [CS1], which also reveals that $N_{2}-2 n(23-n)$ is always a multiple of $2^{4}$ even for $n=22$ and $n=23$; a "conceptual" (but far from easy) proof of this is found in [Bo, Thm. 4.4.2(3)]. Note that even though we have only proved (12) for $n<24$, it in fact holds for all $n$, since $N_{2}$ is always an even integer.

\section{Estimates for self-dual binary codes}

We recall some basic facts about binary linear codes; see for instance [CS1, Ch.3, §2.2]. Let $F=\mathbf{Z} / 2 \mathbf{Z}$ be the two-element field. We work in the 
vector space $F^{n}$, whose elements we regard as "words" of length $n$ whose "letters" are taken from the "alphabet" F. The (Hamming) "weight" $\operatorname{wt}(w)$ of a word $w=\left(w_{1}, w_{2}, \ldots, w_{n}\right) \in F^{n}$ is $\#\left\{j: w_{j}=1\right\}$ of nonzero coordinates of $w$. A "binary linear code" of length $n$ is a subspace $C \subset$ $F^{n}$. A binary self-dual code is a linear code which is its own annihilator under the nondegenerate pairing $(\cdot, \cdot): C \times C \rightarrow F$, defined by $(v, w)=$ $\sum_{j=1}^{n} v_{j} w_{j}$. Such a code must have dimension $n / 2$, and thus can only exist if $n$ is even, which we henceforth assume. Note that under our pairing we have

$$
(w, w)=\left(w, 1^{n}\right) \equiv \operatorname{wt}(w) \bmod 2
$$

for all $w \in F^{n}$, where $1^{n}$ is the all-ones vector in $F^{n}$. Thus if $C$ is a self-dual code then $C \ni 1^{n}$ and all the words in $C$ have even weight.

The "weight enumerator" $W_{C}$ of $C$ is a generating function for the weight distribution of $C$ :

$$
W_{C}(x, y):=\sum_{c \in C} x^{n-\mathrm{wt}(c)} y^{\mathrm{wt}(c)}
$$

For a binary self-dual code, a theorem of Gleason (Thm.6 of [CS1, Ch.7]), analogous to Hecke's theorem for theta series of lattices, states that $W_{C}$ is a weighted-homogeneous polynomial $P_{W}\left(x^{2}+y^{2}, x^{8}+14 x^{4} y^{4}+y^{8}\right)$ in the weight enumerators of the double repetition code $\mathbf{z}:=\{(0,0),(1,1)\} \subset F^{2}$ and the extended Hamming code in $F^{8}$ respectively.

Analogous to the homomorphism $v \mapsto|v|^{2} \bmod 2$ from an integral lattice to $\mathbf{Z} / 2$, we have for any self-dual code $C \subset F^{n}$ a linear map from $C$ to $F$ taking any $c \in C$ to $\frac{1}{2} \operatorname{wt}(c) \bmod 2$. We can use the pairing on $F^{n}$ to represent any linear functional on a self-dual code by a unique coset of the code; thus we find a coset $C^{\prime}$ of $C$ consisting of all $c^{\prime} \in F^{n}$ such that

$$
\frac{1}{2} \mathrm{wt}(c) \equiv\left(c, c^{\prime}\right) \bmod 2
$$

for all $c \in C$. As in [CS2] we call $C^{\prime}$ the shadow of $C$, in analogy with the shadow of an integral unimodular lattice. Let

$$
W_{C}^{\prime}(x, y):=\sum_{c \in C^{\prime}} x^{n-\mathrm{wt}(c)} y^{\mathrm{wt}(c)}
$$

be the generating function for the weight distribution of $C^{\prime}$. Using discrete Poisson inversion as in the proof of the MacWilliams identity, and the 
characterization (15) of $C^{\prime}$, we find as in [CS2] that

$$
\begin{aligned}
W_{C}^{\prime}(x, y) & =2^{-n / 2} \sum_{c \in C}(-1)^{\mathrm{wt}(c) / 2}(x+y)^{n-\mathrm{wt}(c)}(x-y)^{\mathrm{wt}(c)} \\
& =2^{-n / 2} W_{C}(x+y, i(x-y)) .
\end{aligned}
$$

Thus from $W_{C}(x, y)=P_{W}\left(x^{2}+y^{2}, x^{8}+14 x^{4} y^{4}+y^{8}\right)$ we obtain

$$
W_{C}^{\prime}(x, y)=P_{W}\left(2 x y, x^{8}+14 x^{4} y^{4}+y^{8}\right) .
$$

Note that all the words in the shadow thus have weight congruent to $n / 2$ mod 4 . We could have also obtained this directly from the MacWilliams identity

$$
W_{C}(x, y)=2^{-n / 2} W_{C}(x+y, x-y)
$$

(which also underlies Gleason's theorem); this would more closely parallel the analytic proof of $|w|^{2} \equiv n \bmod 8$ in [El].

If $c \in C$ has weight 2 then every codeword either contains or is disjoint from $c$. Thus $C$ decomposes as a direct sum of a double repetition code $\mathbf{z}$ generated by $c$ and the self-dual code of length $n-2$ consisting of codewords disjoint from $c$. Iterating this we decompose $C$ as $C_{0} \oplus \mathbf{z}^{r}$, where $r$ is the number of weight-2 words in $C$, and $C_{0}$ is a self-dual code of length $n-2 r$ with no words of weight 2 . Now for any self-dual codes $C_{1}, C_{2}$, their direct sum $C_{1} \oplus C_{2}$ has shadow

$$
\left(C_{1} \oplus C_{2}\right)^{\prime}=C_{1}^{\prime} \oplus C_{2}^{\prime}
$$

Since the shadow of $\mathbf{z}$ is $\{(0,1),(1,0)\}$, it follows that the shadow of $\mathbf{z}^{r}$ consists entirely of words of weight $r$, and if $C=C_{0} \oplus \mathbf{z}^{r}$ then the minimal weight of $C_{0}^{\prime}$ is $r$ less than that of $C^{\prime}$.

Since $C^{\prime}$ contains $w+1^{n}$ whenever it contains $w$, it is clear that the minimal weight of $C^{\prime}$ cannot exceed the value $n / 2$ attained by $\mathbf{z}^{n / 2}$. This is much easier than proving the corresponding fact for characteristic vectors of unimodular lattices, but it does not show that $\mathbf{z}^{n / 2}$ is the only self-dual code whose shadow has minimal weight $n / 2$. We prove this, as we did for lattices, by noting that such a code $C$ must have $W_{C}^{\prime}(x, y)=(2 x y)^{n / 2}$, whence $W_{C}(x, y)=\left(x^{2}+y^{2}\right)^{n / 2}$. Since $C$ contains $n / 2$ words of weight 2 , then, it can only be $\mathbf{z}^{n / 2}$.

We have shown that the shadow of a binary self-dual code $C$ other than $\mathbf{z}^{n / 2}$ contains some words of weight $<n / 2$. Thus $C^{\prime}$ has minimal weight at most $(n-8) / 2$. We next characterize all $C$ attaining this bound. If $C=C_{0} \oplus \mathbf{z}^{r}$ then $C$ attains the bound if and only if $C_{0}$ does, so we need only consider codes without weight-2 words. 
Theorem 1A. Let $C$ be a binary self-dual code of length $n$ with no codewords of weight 2. Then:

i) $C$ has at least $n(22-n) / 8$ codewords of weight 4 .

ii) Equality holds if and only if the shadow of $C$ contains no codewords of weight $<(n-8) / 2$.

iii) In that case the number of codewords of weight exactly $(n-8) / 2$ in the shadow is $2^{(n-14) / 2} n$.

Proof. We can mimic the proof of Theorem 1. If the minimal weight of $C^{\prime}$ is at least $(n-8) / 2$ then $W_{C}^{\prime}$ is a linear combination of $(x y)^{n / 2}$ and $(x y)^{(n-8) / 2}\left(x^{8}+14 x^{4} y^{4}+y^{8}\right)$, and thus $W_{C}(x, y)$ is a linear combination of $\left(x^{2}+y^{2}\right)^{n / 2}$ and $\left(x^{2}+y^{2}\right)^{(n-8) / 2}\left(x^{8}+14 x^{4} y^{4}+y^{8}\right)$. The condition that $C$ have no weight-2 codewords then forces

$$
\begin{aligned}
W_{C}(x, y)= & \left(x^{2}+y^{2}\right)^{n / 2} \\
& -\frac{n}{8}\left(x^{2}+y^{2}\right)^{(n-8) / 2}\left(\left(x^{2}+y^{2}\right)^{4}-\left(x^{8}+14 x^{4} y^{4}+y^{8}\right)\right) \\
= & x^{n}+0 x^{n-2} y^{2}+\frac{n(22-n)}{8} x^{n-4} y^{4}+\cdots
\end{aligned}
$$

and

$$
W_{C}(x, y)=(2 x y)^{n / 2}-\frac{n}{8}(2 x y)^{(n-8) / 2}\left[(2 x y)^{4}-\left(x^{8}+14 x^{4} y^{4}+y^{8}\right)\right]
$$

$$
=2^{(n-14) / 2}(x y)^{(n-8) / 2}\left[n x^{8}+(128-2 n) x^{4} y^{4}+n y^{8}\right] .
$$

If $n<24$ and $C$ is any binary self-dual code of length $n$ containing no words of weight 2 and $n(22-n) / 8+d$ words of weight 4 then its weight enumerator exceeds (21) by

$$
\frac{d}{16}\left(x^{2}+y^{2}\right)^{(n-16) / 2}\left[x^{8}+14 x^{4} y^{4}+y^{8}-\left(x^{2}+y^{2}\right)^{4}\right]^{2},
$$

so the weight enumerator of the shadow $C^{\prime}$ exceeds (22) by

$$
\frac{d}{16}(2 x y)^{(n-16) / 2}\left(x^{8}-2 x^{4} y^{4}+y^{8}\right)^{2} .
$$

Thus $C^{\prime}$ contains $2^{(n-24) / 2} d$ words of weight $(n-16) / 2$, from which we find that $d$ is a nonnegative multiple of $2^{(24-n) / 2}$.

Alternatively we could deduce Theorem 1A from Theorem 1 via Construction A [CS1, Ch.7, §2]. Recall that this construction associates to a self-dual code $C \subset F^{n}$ the unimodular integral lattice

$$
L_{C}:=\left\{2^{-1 / 2} v \mid v \in \mathbf{Z}^{n}, v \bmod 2 \in C\right\} .
$$


The theta series of this lattice is given by

$$
\theta_{L}(t)=W_{C}\left(\theta_{\mathbf{Z}}(2 t), \theta_{\mathbf{Z}}^{\prime}(2 t)\right) .
$$

In particular, $L_{C}$ has no vectors of norm 1 if and only if $C$ has no codewords of weight 2 (NB $L_{\mathbf{z}} \cong \mathbf{Z}^{2}$ ), and the $N_{2}\left(L_{C}\right)-2 n$ is $2^{4}$ times the number of weight-4 codewords in $C$. Moreover the set of characteristic vectors of $L_{C}$ is

$$
\left\{2^{1 / 2} v \mid v \in \mathbf{Z}^{n}, v \bmod 2 \in C^{\prime}\right\}
$$

(in effect the shadow of $L_{C}$ is obtained by applying Construction A to the shadow of $C$ ), so the norm of the shortest characteristic vectors is half the minimal weight of $C^{\prime}$. Thus applying Theorem 1 to $L_{C}$ yields Theorem $1 \mathrm{~A}$ immediately.

The codes $C$ of parts (ii), (iii) of Theorem $1 \mathrm{~A}$ are precisely those for which $L_{C}$ is one of the 14 lattices listed in connection with Theorem 1. Of course, not every such lattice arises, because $n$ must be even. Moreover, the root system can only involve $A_{1}, D_{2 m}, E_{7}$ and $E_{8}$ if the lattice arises from construction $A$. This leaves only the seven lattices with root systems $E_{8}, D_{12}, E_{7}^{2}, D_{8}^{2}, D_{6}^{3}, D_{4}^{5}, A_{1}^{22}$ of rank $8,12,14,16,18,20,22$ respectively. It turns out that each of those lattices arises as $L_{C}$ for a unique code $C$ [Pl, PS]. For instance, the first of these arises from the extended Hamming code, and the last from what might be called the shorter binary Golay code; these are the shortest self-dual binary codes having minimal weight 4 and 6 respectively. Again it so happens that whenever there is a self-dual code of length $n<24$ with minimal weight at least 4 , there is such a code (this time unique) with only $n(22-n) / 8$ words of weight 4 , so Conway's description of our fourteen lattices also applies mutatis mutandis to our seven codes.

\section{Can we go past $n-8$ ?}

Our results suggest the following questions:

For any $k>0$, is there $N_{k}$ such that every integral unimodular lattice all of whose characteristic vectors have norm $\geq n-8 k$ is of the form $L_{0} \oplus \mathbf{Z}^{r}$ for some lattice $L_{0}$ of rank at most $N_{k}$ ?

For any $k>0$, is there $n_{k}$ such that every binary self-dual code of whose shadow has minimal norm $\geq(n-8 k) / 2$ is of the form $C_{0} \oplus \mathbf{z}^{r}$ for some code $C_{0}$ of length at most $n_{k}$ ?

Of course, a positive answer for lattices would imply one for codes, and vice versa for a negative answer, via Construction A, with $n_{k} \leq N_{k}$ in the former case. Even $k=2$ seems difficult. 


\section{Acknowledgement}

Thanks to John H. Conway for helpful correspondence.

\section{References}

[Bo] R. Borcherds, The Leech lattice and other lattices, Ph.D. thesis, Cambridge (Trinity College), 1984.

[CS1] J. H. Conway and N. J. A. Sloane, Sphere Packings, Lattices and Groups, Springer, New York, 1993.

[CS2] _ A new upper bound on the minimal distance of self-dual codes, IEEE Trans. Inform. Theory 36 (1990), 1319-1333.

[El] N. D. Elkies, A characterization of the $\mathbf{Z}^{n}$ lattice, Math. Research Letters 2 (1995), 321-326.

[Pl] V. Pless A classification of self-orthogonal codes over $G F(2)$, Discr. Math 3 (1972), 209-246.

[PS] V. Pless and N. J. A. Sloane, On the classification and enumeration of self-dual codes, Comb. Th A18 (1975), 313-335.

[Se] J. P. Serre, A Course in Arithmetic, Springer, New York, 1973.

Department of Mathematics, Harvard University, Cambridge MA 02138

E-mail address: elkies@zariski.harvard.edu 